\title{
Radioanatomic Characteristics of the Posteromedial Intraconal Space: Implications for Endoscopic Resection of Orbital Lesions
}

\author{
(D) A. Jafari, (D) A.E. Lehmann, (D) N. Wolkow, (D)A.F. Juliano, (D)B.S. Bleier, and (D) K.L. Reinshagen
}

\begin{abstract}
BACKGROUND AND PURPOSE: Imaging is essential in the diagnostic work-up of patients with orbital lesions. The position of an orbital lesion relative to the inferomedial muscular trunk of the ophthalmic artery determines endoscopic resectability, anticipated technical difficulty, and patient morbidity. Although the inferomedial muscular trunk is not readily identifiable on preoperative imaging, we hypothesize that it is spatially approximate to the location where the ophthalmic artery crosses the optic nerve. Our aim was to determine whether the ophthalmic artery-optic nerve crosspoint anatomically approximates the inferomedial muscular trunk in a cadaver study and can be appreciated on imaging of known posteromedial orbital lesions.
\end{abstract}

MATERIALS AND METHODS: Dissection was performed on 17 fresh-frozen cadaver orbits to assess the relationship between the inferomedial muscular trunk and ophthalmic artery-optic nerve crosspoint. Retrospective review of imaging in 9 patients with posteromedial orbital lesions assessed posteromedial orbital compartment characteristics and the ability to locate the ophthalmic artery-optic nerve crosspoint.

RESULTS: In our cadaver study, the mean distance between the ophthalmic artery-optic nerve crosspoint and the inferomedial muscular trunk was $1.21 \pm 0.64 \mathrm{~mm}$. Retrospectively, the ophthalmic artery-optic nerve crosspoint was identifiable in $9 / 9$ patients, whereas the inferomedial muscular trunk was not identifiable in any patient. Total or partial effacement of the posteromedial intraconal fat triangle was observed in $9 / 9$ patients.

CONCLUSIONS: This study of neurovascular relationships within the posteromedial orbit demonstrates that the ophthalmic arteryoptic nerve crosspoint closely approximates the inferomedial muscular trunk and can be seen in patients with posteromedial orbital lesions. Posteromedial intraconal fat effacement may help to localize these lesions. These findings may facilitate multidisciplinary communication and help predict lesion resectability and patient outcomes.

ABBREVIATIONS: $\mathrm{CECT}=$ contrast-enhanced $\mathrm{CT} ; \quad \mathrm{IMT}=$ inferomedial muscular trunk of the ophthalmic artery; $\mathrm{NECT}=$ non-contrast-enhanced $\mathrm{CT} ; \mathrm{OA}=$ ophthalmic artery; $\mathrm{ON}=$ optic nerve

$\mathbf{R}$ adiologic characteristics of orbital lesions as well as their locations relative to critical neurovascular structures are essential to determine the surgical approach for resection and to anticipate outcomes. ${ }^{1-3}$ A keen understanding of orbital anatomy is required to determine whether an orbital lesion is amenable to endoscopic

Received April 27, 2020; accepted after revision July 29.

From the Departments of Otolaryngology-Head \& Neck Surgery (A.J., A.E.L., B.S.B.), Ophthalmology (N.W.), and Radiology (A.F.., K.L.R.), Massachusetts Eye and Ear, Harvard Medical School, Boston, Massachusetts; and Department of OtolaryngologyHead \& Neck Surgery (A.J.), University of Washington, Seattle, Washington.

Paper previously presented at: Annual Meeting of the American Rhinologic Society, September 11-12, 2020; Virtual.

Please address correspondence to Aria Jafari, MD, Division of Rhinology and Endoscopic Skull Base Surgery, Department of Otolaryngology-Head \& Neck Surgery, University of Washington, Box 356515, Health Sciences Building, Suite BB1165, Seattle, WA 98195; e-mail: ajafari@uw.edu

Indicates article with supplemental on-line table.

http://dx.doi.org/10.3174/ajnr.A6822 resection and which structures are at risk. However, certain orbital structures that are essential to surgical planning and that determine surgical complexity are not readily discernable on imaging.

For example, the inferomedial muscular trunk (IMT) of the ophthalmic artery (OA) defines the anterior and posterior medial compartments of the orbit when considering an endoscopic resection. ${ }^{2,3}$ Injury of the IMT may result in bleeding and ischemia of the medial and inferior rectus muscles, which can cause retrobulbar hemorrhage, difficult/incomplete resection, or diplopia. $^{3}$ Thus, lesions located posterior to the IMT tend to be more difficult to resect and have a greater risk of poorer patient outcomes. ${ }^{3}$ Thus, the position of a lesion relative to the IMT is an important component of the validated Cavernous Hemangioma Exclusively Endonasal Resection orbital staging system. ${ }^{3}$

Although the IMT is a key landmark for operative planning and surgical dissection, it has a relatively small caliber (approximately 
$0.7 \mathrm{~mm})^{4}$ and is, thus, challenging to identify on preoperative imaging. Prior anatomic studies have described the IMT taking off from the inferior aspect of the OA near its second bend. ${ }^{5}$ The location of this bend is thought to spatially approximate the location where the OA crosses the optic nerve (ON) at the medial aspect of the nerve (the OA-ON crosspoint). Although the IMT is not reliably seen on imaging, the OA-ON crosspoint is a radiologically salient finding. ${ }^{4,5}$ However, the precise anatomic relationship of the OA-ON crosspoint and the IMT has not yet been investigated.

Reporting the OA-ON crosspoint may enhance multidisciplinary communication between neuroradiologists and orbital surgeons. The aim of this study was to determine the following: 1) whether the OA-ON crosspoint anatomically approximates the IMT in a cadaver study, and 2) whether this radiologic landmark (the OA-ON crosspoint) could be appreciated on imaging of known orbital lesions. We posit that this is a readily identifiable radiologic landmark in cases of orbital lesions.

\section{MATERIALS AND METHODS}

This study consisted of 2 parts: The first part was an anatomic evaluation using cadaveric specimens to determine the neurovascular relationships between the $\mathrm{OA}$ and $\mathrm{ON}$, including the OAON crosspoint. The second part of the study assessed whether this OA-ON crosspoint could be identified on preoperative imaging in a retrospective case series of posteromedial orbital lesions. The study was approved by the Institutional Review Board of Partners HealthCare, Protocol No. 2019P003408.

\section{Anatomic Evaluation}

Anatomic dissection was performed on 9 fresh-frozen cadaver head specimens ( 8 without latex injection and 1 with latex injection). One orbit was not included because this specimen had a prior enucleation. Two included specimens (totaling 4 orbits) had their corneas donated and were thus missing the anterior segments of their globes. A total of 17 orbits were dissected. Three specimens were male and 6 were female. The mean age at death was $63.2 \pm$ 16.5 years (range, $39-86$ years).

For each specimen, a bicoronal incision was performed extending from the zygomatic roots through the periosteum and down to cranial bone. A soft-tissue flap was elevated anteriorly to the level of the supraorbital ridges, and the frontal bone was exposed. A bifrontal craniotomy was planned, and a bone flap was created using a high-powered drill. After elevation of the bone flap from the dura, a durotomy was performed, and the frontal lobe contents were removed. The dura and remaining intracranial contents were then dissected off the cribriform plate in an anterior-to-posterior fashion to expose the orbital roofs bilaterally. An osteotomy was created in the orbital roof, and rongeurs were used to remove the bone of the orbital roof and expose the periorbita. The periorbita was then incised, and the orbital contents were visualized. This portion of the dissection was performed by 2 otolaryngologists (A.J. and A.E.L.).

Once the periorbita was opened, the frontal nerve, levator palpebrae superioris, and superior rectus muscles were transected anteriorly and reflected posteriorly. Orbital fat was removed to reveal the $\mathrm{ON}$ and the OA. The branches of the OA were dissected free of orbital fat and followed to their targets. In all

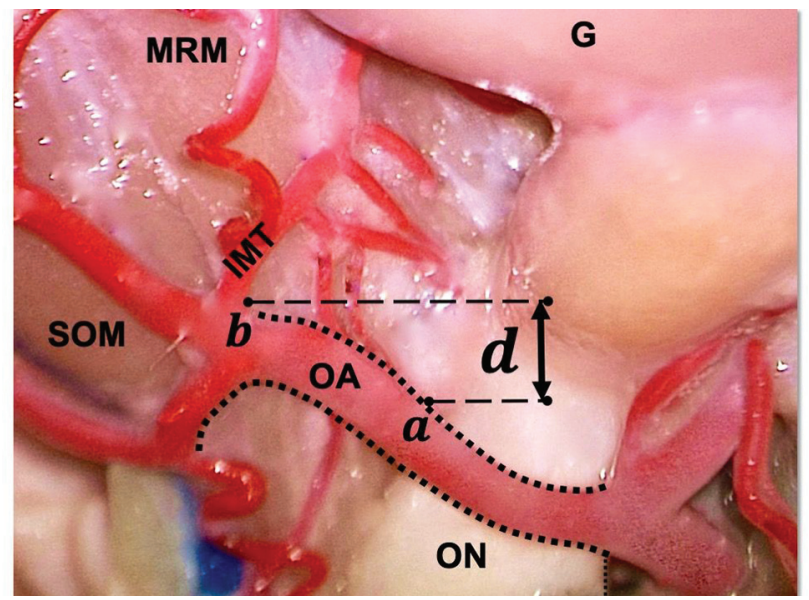

FIG 1. Dorsal view of the right orbit and its contents, with the medial rectus muscle (MRM) and superior oblique muscle (SOM) reflected laterally. G indicates globe; a, OA-ON cross-point; b, branch point of IMT; $d$, distance (in millimeters) from the OA-ON crosspoint and the IMT branch point.

specimens, the OA emerged from the optic canal lateral to the $\mathrm{ON}$. The OA then crossed above the ON, coursing along the medial aspect of the $\mathrm{ON}$ in the posteromedial intraconal space. In all specimens, the IMT emerged from the inferior aspect of the $\mathrm{OA}$ and was identified medial to the ON, with its branches heading toward the medial and inferior rectus muscles. This portion of the dissection was performed by an ophthalmologist (N.W.).

Quantitative measurements were then obtained. The OA was traced to the point at which the vessel crossed the medial aspect of the ON, and this crosspoint was marked. The distance between this crosspoint and the point of origin of the IMT from the OA was measured using calipers, and digital still images illustrating the surgical relationships were captured (see Fig 1 for illustration of these points and orientation of distance measurement). All 3 investigators involved in the dissections participated in obtaining the distance measurements for all orbits (A.J., A.E.L., and N.W.).

\section{Radiographic Evaluation}

We reviewed 9 consecutive patients with posterior intraconal orbital pathology who had undergone both MR and CT orbital imaging. Seven of 9 patients underwent 3T MR imaging at our institution, while 2 patients had outside 1.5T MRIs reviewed. Seven of 9 patients underwent $\mathrm{CT}$ at our institution, while 2 patients had outside CT studies reviewed. All MRIs were performed at our institution on the same 3T Achieva scanner with an 8-channel head coil (Philips Healthcare). Eight of 9 patients had intravenous gadolinium-based contrast, while 1 patient could not receive MR imaging contrast due to prior anaphylaxis. All MRIs performed included 2to 3-mm section thickness, precontrast axial \pm coronal T1WI, and postcontrast axial \pm coronal T1WI of the orbits with or without fat suppression. At 3T, T1WI was performed with TE $=10-12 \mathrm{~ms}$, $\mathrm{TR}=510-710 \mathrm{~ms}$, number of excitations $=1-2$, flip angle $=90^{\circ}$, axial FOV $=140 \times 140 \mathrm{~mm}$, coronal FOV $=150 \times 150 \mathrm{~mm}$, axial bandwidth $=163-170 \mathrm{~Hz}$, coronal bandwidth $=246-300 \mathrm{~Hz}$, acquired pixel size in the axial plane $=0.43 \times 0.43 \mathrm{~mm}$, acquired pixel size in the coronal plane $=0.47 \times 0.47 \mathrm{~mm}$, scan time 
(minute:second) $=$ axial $(2-3 \mathrm{~mm}): 4$ minutes and 21 seconds and coronal ( $3 \mathrm{~mm}): 4$ minutes and 11 seconds. Outside 1.5T T1WI was performed with $\mathrm{TE}=10-17 \mathrm{~ms}$, TR $=492-635 \mathrm{~ms}$, number of excitations $=1-3$, flip angle $=90^{\circ}$.

All MR images evaluated were 2D. All CTs were performed on 64-section multidetector units. Of those performed at our institution, all were performed on the same Discovery 750HD scanner (GE Healthcare). All CTs were performed with $120 \mathrm{kV}$ and had a range of 60-258 mA. Three of 9 patients underwent contrast-enhanced CT (CECT), while the remainder (6/9) underwent non-contrastenhanced CT (NECT). CT images were reviewed in 2- to 3-mm section-thickness axial and coronal reformats in a soft-tissue window. The images were reviewed independently by 2 attending neuroradiologists (A.F.J. and K.L.R., with 12 and 4 years' experience, respectively), who determined, by consensus, the visibility of the OA-ON crosspoint on $\mathrm{CT}$ and MR imaging. The OA-ON crosspoint was assessed with axial 2D-T1WI spin-echo sequences and, if available, axial or coronal 2D-T1WI fat-suppressed postcontrast images.

Because posteromedial orbital lesions are considered more difficult to resect than their anteromedial counterparts, we also assessed whether orbital fat effacement in the posteromedial intraconal triangle was a radiologic characteristic in this series of posteromedial orbital lesions. The effacement of orbital fat within the posteromedial intraconal triangle, defined anteriorly by a coronally oriented line through the OA-ON crosspoint, laterally by the long axis of the medial rectus, medially by the $\mathrm{ON}$, and posteriorly by the orbital apex, was determined on precontrast T1WI or CT images. The degree of orbital fat effacement was determined subjectively as follows: At the level of the mass, no visible fat was described as completely effaced, and $>50 \%$ degree of fat effacement was described as near-total or partial fat effacement. The neuroradiologists were blinded to the results of the cadaveric dissection and intraoperative findings.

\section{RESULTS}

\section{Anatomic Neurovascular Relationships}

In our cadaver study, the OA was encountered lateral to the $\mathrm{ON}$ after exiting the optic canal in all (17/17) orbits. The course of the OA then proceeded from lateral to medial. The OA traversed above the ON in all (17/17) orbits. The point at which the OA crossed the medial aspect of the ON (point a) and the IMT (point b) was able to be identified in all (17/17) specimens, as seen in Fig 1. The IMT was located proximal to the OA-ON crosspoint in 1 orbit. The mean distance between these 2 landmarks (point a to b) was determined to be $1.31 \pm 0.75 \mathrm{~mm}$ on the left, $1.11 \pm 0.55 \mathrm{~mm}$ on the right $(P=.53)$, and $1.21 \pm 0.64 \mathrm{~mm}$ for all orbits combined. Any discrepancy in measurements was resolved by consensus by investigators (A.J., A.E.L, N.W.).

\section{Radiologic Characteristics}

In our retrospective case series of patients with orbital apex lesions, 3 patients were men and 6 were women. The mean age was $48.9 \pm$ 22.2 years. Dates of imaging were from November 2012 to September 2019. Most lesions were orbital venous malformations, previously referred to as cavernous hemangiomas (5/9, 55.6\%), and the remainder were rhabdomyosarcoma, meningioma, squamous cell carcinoma, and schwannoma (1 each). The OA was visualized

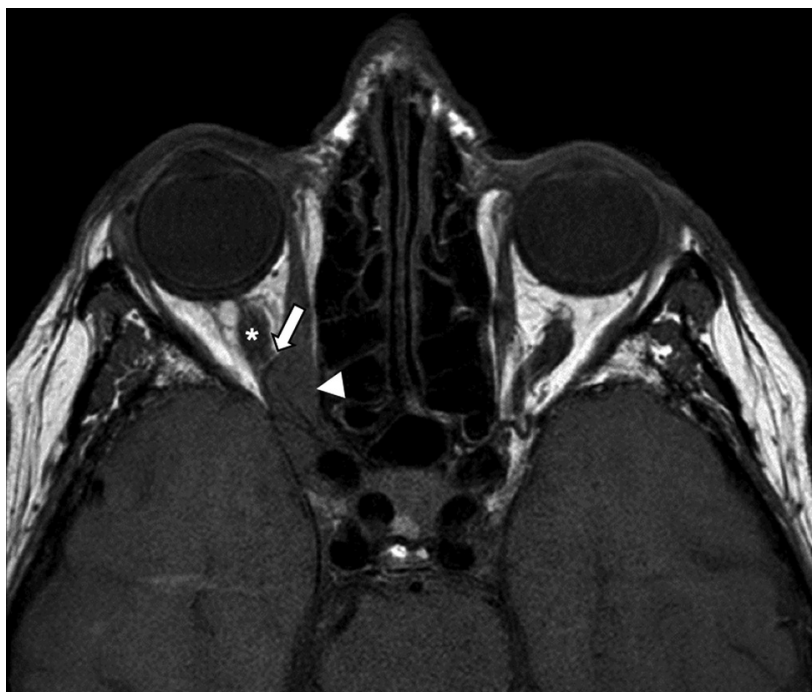

FIG 2. Axial T1-weighted MR image in a 75-year-old woman with squamous cell carcinoma (white arrowhead) in the posterior intraconal right orbit. The OA (white arrow) is seen crossing the medial margin of the ON (asterisk).

lateral to the $\mathrm{ON}$ after exiting the optic canal, traversing from lateral to medial in all (9/9) orbits. Additional details are shown in the Online Table. The point at which the OA crossed the medial aspect of the ON (the OA-ON crosspoint) could be identified on imaging for all (9/9) patients (Figs 2-5). The OA-ON crosspoint was visible on non-fat-suppressed T1WI in all (9/9) patients. While 8 of 9 patients had fat-suppressed, postcontrast T1WI of the orbits, the OA-ON crosspoint was only visible in 2 of 8 patients in these fatsuppressed sequences. Of the 6 patients who underwent NECT, the OA-ON crosspoint could be seen in 6 patients (100\%) (Fig $3 A-C$ ). Of the 3 patients who underwent CECT, the OA-ON crosspoint could be seen in 2 patients (66.7\%) (Fig 4). The IMT could not be identified in any (0/9) of the imaging studies. The posteromedial intraconal fat triangle characteristics were recorded and classified into categories based on the degree of effacement by the lesion. Near-total or partial effacement was observed in 3 of 9 (33.3\%) patients (Fig 3), and total effacement was observed in 6 of 9 (67.7\%) patients (Fig 5).

\section{DISCUSSION}

Diagnostic imaging and interpretation are essential in the workup of patients with orbital lesions, especially because the physical examination may be unremarkable early in the disease. ${ }^{1}$ The precise location of an orbital lesion within the orbit and its position relative to the IMT of the OA determine endoscopic resectability, anticipated technical difficulty, and patient morbidity. ${ }^{2,3,6}$ This feature is most likely relevant for solitary lesions with well-circumscribed, pushing borders as opposed to infiltrative lesions in which the IMT may be engulfed by the lesion. However, despite its significance during orbital lesion surgery, the IMT is not readily identifiable on preoperative diagnostic imaging.

The purpose of this study was to integrate neurovascular associations, radiologic findings, and surgical anatomy to describe the posteromedial orbit, a highly complex and technically challenging space. Our results demonstrate that the OA-ON crosspoint is a

AJNR Am J Neuroradiol 41:2327-32 Dec 2020 www.ajnr.org 

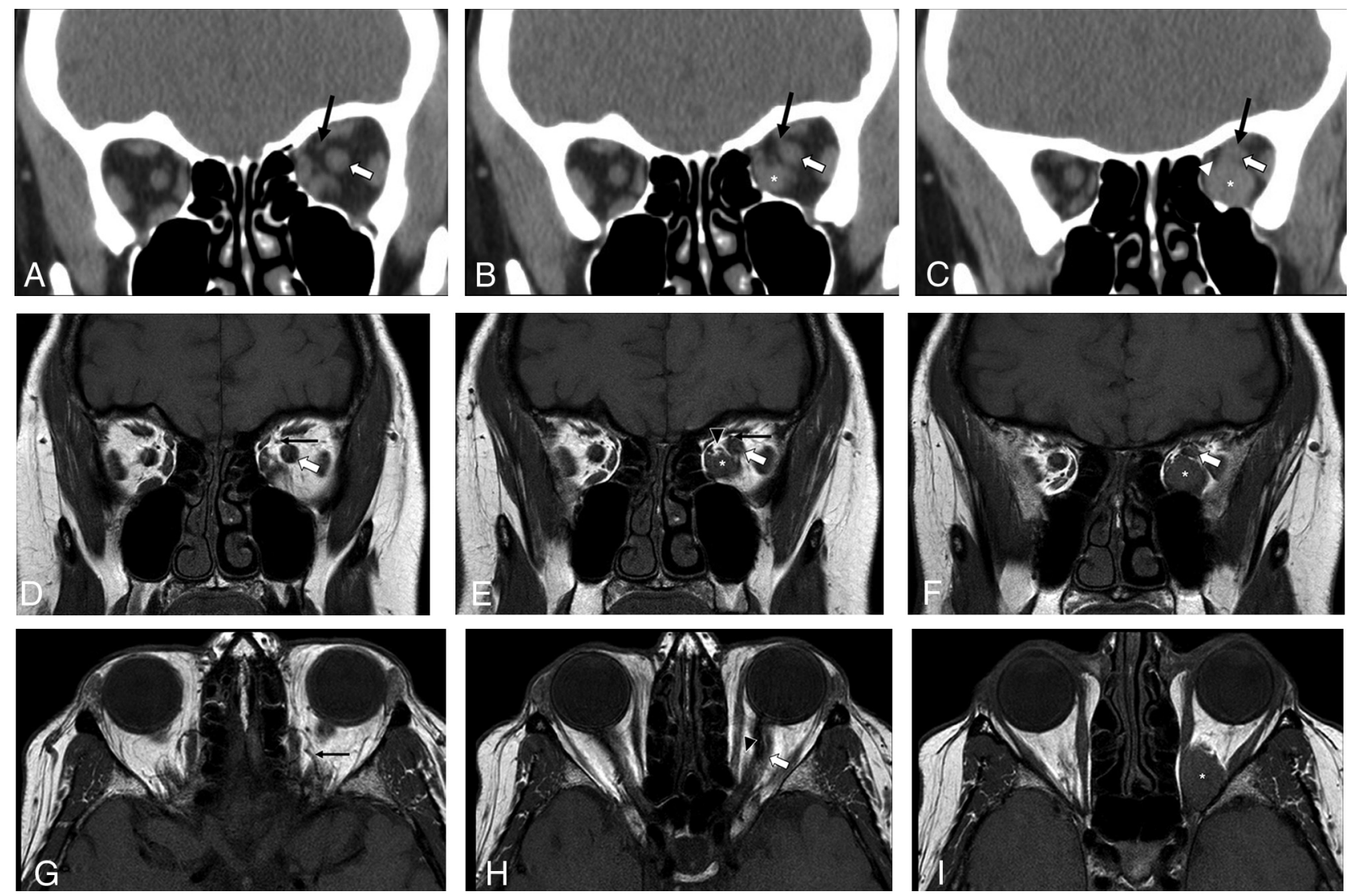

FIG 3. Orbital imaging in a 69-year-old woman with a venous malformation in the posterior intraconal left orbit (asterisk). Coronal NECT images ( $A-C$, anterior to posterior) show the OA (black arrow) crossing the medial margin of the ON (white arrow, $B$ ). There is incomplete effacement of the fat at the level of the lesion within the posteromedial intraconal space on CT (white arrowhead). Coronal T7-weighted (D-F, anterior to posterior) and axial T1-weighted MR images (G-l, superior to inferior) of the orbits show the OA (black arrow) coursing above the ON (white arrow). The OA is seen crossing the medial margin of the ON (E). There is incomplete effacement of the posteromedial intraconal fat on MR imaging at the level of the lesion (black arrowhead).
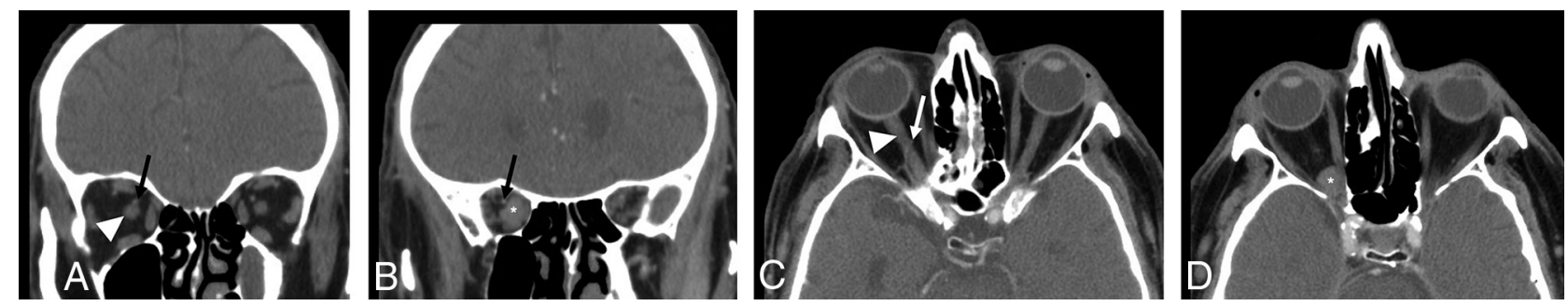

FIG 4. CECT of the orbits in a 74-year-old man with a venous malformation in the posterior intraconal right orbit. Coronal images ( $A$ and $B$, anterior to posterior) demonstrate the OA (black arrow) crossing the medial margin of the ON (white arrowhead, $A$ ) anterior to the lesion (asterisk). Axial images ( $C$ and $D$, superior to inferior) also demonstrate the OA (white arrow) crossing the medial margin of the ON (white arrowhead, $C$ ) anterior to the lesion (asterisk, D). Effacement of the fat in the posteromedial intraconal space is demonstrated at the level of the lesion $(D)$.

radiologically consistent finding on our retrospective imaging review, and it closely approximates the location of the origin of the IMT from the OA (mean distance, $1.21 \pm 0.64 \mathrm{~mm}$ ) on our cadaveric study. In addition, we found that effacement of the posteromedial intraconal fat triangle on imaging was seen in our select patient sample with pathology within this space. These radioanatomic findings offer additional tools for effective reporting because endoscopic orbital surgery is used in an increasingly broad array of orbital pathologies.
Findings from previous anatomic dissections of neurovascular structures detail the close association of the $\mathrm{OA}$ and $\mathrm{ON}$ within the posterior orbit and support the findings demonstrated in this study. ${ }^{4-6}$ In the optic canal and the posterior orbit, the OA is adherent to the ON through connective tissue attachments to the ON dural sheath. ${ }^{5}$ This adherence extends throughout the optic canal and into the posterior orbit as the OA crosses the ON from lateral to medial. ${ }^{5}$ After the OA crosses the medial margin of the $\mathrm{ON}$, the OA turns anteriorly, gives off the IMT, and is then no 

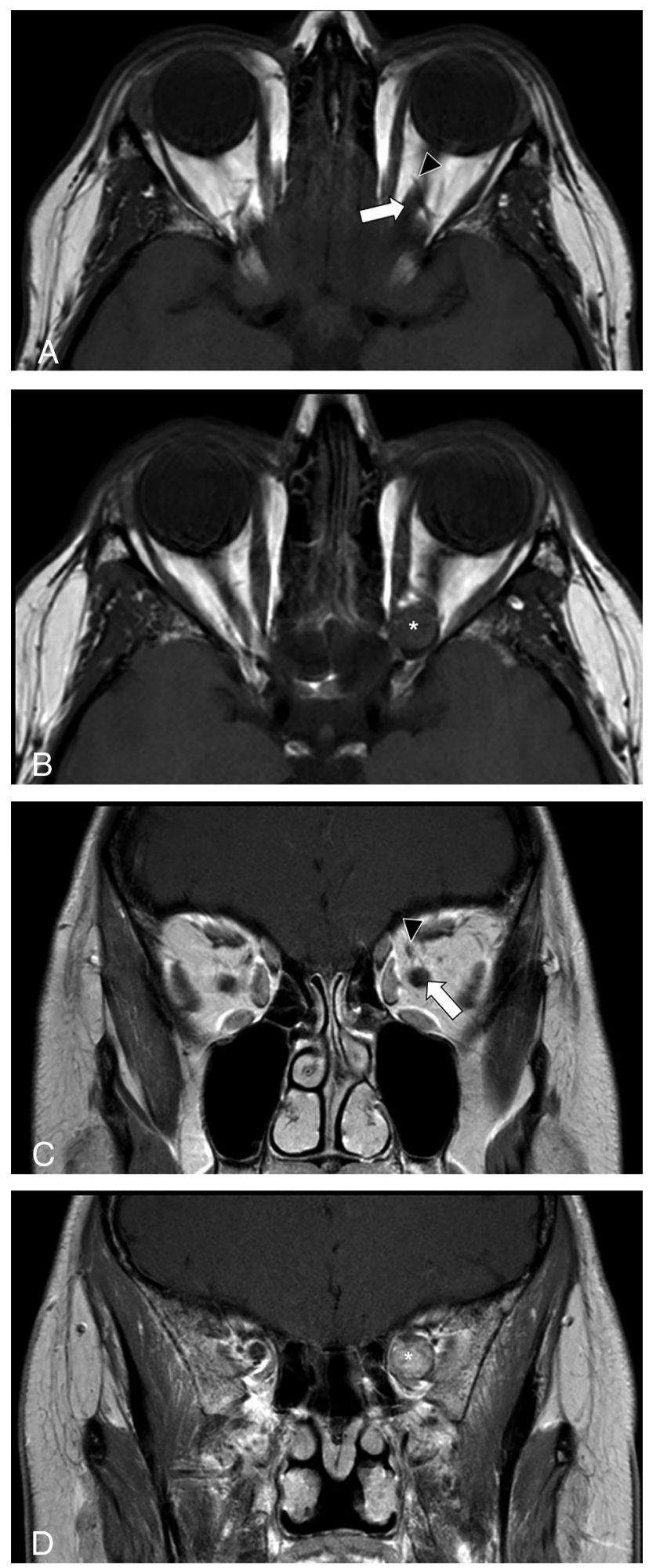

FIG 5. Axial T7-weighted precontrast ( $A$ and $B$, superior to inferior) and coronal Tl-weighted postcontrast ( $C$ and $D$, anterior to posterior) images of the orbits in a 39-year-old woman with a venous malformation in the posterior intraconal left orbit. The OA (black arrowhead) can be seen crossing the medial margin of the ON (white arrow, $A$ and $C$ ) anterior to the lesion (asterisk). There is full effacement of the posterior intraconal fat at the level of the lesion ( $B$ and $D)$. longer adherent to the ON. ${ }^{4,5}$ The reliability of the OA-ON crosspoint demonstrated in the present study, along with the known adherence of the OA to the ON in this location, supports the utility of the OA-ON crosspoint in cases of orbital pathology, even when mass effect may distort other soft-tissue landmarks.

Identification of the OA-ON crosspoint (a radiologically distinct structure) as an approximation of the IMT (a radiologically indistinct structure) allows more accurate preoperative staging of orbital lesions, which, in turn, optimizes surgical planning and patient counseling. ${ }^{2,3,7}$ Lesions located posterior to the IMT may require a more complex operation, including binarial access, a 2surgeon approach, and vascularized reconstruction. Lesions in this location may also carry a greater risk for postoperative visual and cosmetic morbidity. ${ }^{3}$ Our study supports the complementary use of orbital MR imaging in the preoperative setting to best visualize the OA-ON crosspoint. While CT is more readily available and often used in intraoperative guidance, the OA-ON crosspoint was not visualized in 1 patient with CECT. In this patient, the OA was similar in density to the avidly enhancing rhabdomyosarcoma and was not definitively visualized at the medial margin of the $\mathrm{ON}$. The $\mathrm{OA}$ may be more visible on MR imaging because the OA in our patients was seen as a dark flow void on precontrast T1WI and thus was more visible against the lesion background and adjacent optic nerve, whereas it typically has a similar density to the surrounding lesion or optic nerve on CT. In addition, the OA-ON crosspoint was best visualized on non-fat-suppressed spin-echo T1WI, likely due to the contrast between the dark flow void of the OA against intraconal orbital fat. This effect is likely due to the black-blood effect seen with spin-echo T1WI and would be expected to be spoiled using a gradient-echo T1WI. This blackblood effect has been effective in vessel wall imaging., 8

In our population with lesions in the posterior intraconal space, we found that lesions that were posterior to the IMT demonstrated partial or complete effacement of the posteromedial fat triangle, which could be a useful additional sign, particularly if the OA-ON crosspoint cannot be visualized. This behavior of fat relative to critical structures is also seen in parapharyngeal space lesions, in which poststyloid lesions demonstrate anterolateral displacement of the prestyloid fat and effacement of the fat around the great vessels. ${ }^{10}$ Because the orbital lesions are similarly space-occupying, we suspect that the effacement seen within the posteromedial orbital compartment reflects similar displacement within the orbit.

This study has limitations. The relatively small sample size of cadaver specimens may have limited our ability to capture anatomic variability inherent in orbital vasculature. For example, in a small subset of patients, the OA may run under the ON, a relationship not seen in our dissections. ${ }^{4,5}$ Furthermore, distance measurements may have been affected by postmortem changes and the inherent compliance of the structures within the mobile orbital fat. In addition, due to the retrospective nature of this study, the MR imaging and CT techniques used varied slightly among patients because outside images were included in our analysis. A prospective study with larger patient numbers to confirm these findings and determine the most efficient imaging strategy for these patients would be helpful. As a result of the retrospective nature of this study, the origin of the 
IMT in relationship to the OA-ON crosspoint was not confirmed intraoperatively in these patients, a further limitation, which would benefit from further prospective studies. In addition, future studies could include the use of high-resolution microscopy coil MR imaging for further characterization of the intraconal orbital anatomy. ${ }^{11}$ Last, further studies are needed to determine whether the identification of the OA-ON crosspoint is linked with decreased morbidity and improved patient outcomes in clinical practice.

\section{CONCLUSIONS}

This study of neurovascular relationships within the posteromedial orbit demonstrates that the OA-ON crosspoint (a radiologically distinct structure) closely approximates the IMT (a radiologically indistinct structure), which is a critical structure for operative planning and surgical dissection of orbital lesions. Partial and complete posteromedial intraconal fat effacement was seen in our patient population with posterior intraconal space lesions. Together, these radiologic findings may facilitate multidisciplinary communication between neuroradiologists and orbital surgeons and help predict lesion resectability and patient outcomes.

Disclosures: Natalie Wolkow-UNRELATED: Consultancy: Pykus Therapeutics, Comments: consulting services on a subject not related to the above work. Benjamin S. Bleier-UNRELATED: Consultancy: Olympus, Karl Storz SE \& Co, Medtronic, Sinopsys, Baxter Healthcare, 3D Matrix; Grants/Grants Pending: 1 R01 NS108968-01 National Institutes of Health/National Institute of Neurological Disorders and Stroke (Principal Investigator), paid to the institution; Royalties: Thieme, paid to the individual. Amy F. Juliano-UNRELATED: Employment: Massachusetts Eye and Ear, Comments: This is my regular full-time job as a practicing clinical radiologist.

\section{REFERENCES}

1. Bilaniuk LT. Orbital vascular lesions: role of imaging. Radiol Clin North Am 1999;37:169-83.xi CrossRef Medline

2. Bleier BS, Healy DY, Chhabra N, et al. Compartmental endoscopic surgical anatomy of the medial intraconal orbital space. Int Forum Allergy Rhinol 2014;4:587-91 CrossRef Medline

3. El Rassi E, Adappa ND, Battaglia P, et al. Development of the international orbital Cavernous Hemangioma Exclusively Endonasal Resection (CHEER) staging system. Int Forum Allergy Rhinol 2019;9:804-12 CrossRef Medline

4. Erdogmus S, Govsa F. Arterial vascularization of the extraocular muscles on its importance for orbital approaches. J Craniofac Surg 2007;18:1125-32 CrossRef Medline

5. Hayreh SS. Orbital vascular anatomy. Eye (Lond) 2006;20:1130-44 CrossRef Medline

6. Li L, London NR Jr, Silva S, et al. Transnasal prelacrimal approach to the inferior intraconal space: a feasibility study. Int Forum Allergy Rhinol 2019;9:1063-68 CrossRef Medline

7. Maxfield AZ, Brook CD, Miyake MM, et al. Compartmental endoscopic surgical anatomy of the inferior intraconal orbital space. $J$ Neurol Surg B Skull Base 2018;79:189-92 CrossRef Medline

8. Mandell DM, Mossa-Basha M, Qiao Y, et al; Vessel Wall Imaging Study Group of the American Society of Neuroradiology. Intracranial vessel wall MRI: principles and expert consensus recommendations of the American Society of Neuroradiology. AJNR Am J Neuroradiol 2017;38:218-29 CrossRef Medline

9. Mohammed-Brahim N, Clavel G, Charbonneau F, et al. Three Tesla 3D high-resolution vessel wall MRI of the orbit may differentiate arteritic from nonarteritic anterior ischemic optic neuropathy. Invest Radiol 2019;54:712-18 CrossRef Medline

10. Stambuk HE, Patel SG. Imaging of the parapharyngeal space. Otolaryngol Clin North Am 2008;41:77-101 CrossRef Medline

11. Dobbs NW, Budak MJ, White RD, et al. MR-Eye: high-resolution microscopy coil MRI for the assessment of the orbit and periorbital structures, Part 1: technique and anatomy. AJNR Am J Neuroradiol 2020;41:947-50 CrossRef Medline 